\title{
A Preliminary Ray Tracing Approach to Computational Electromagnetics for Reverberation Chambers
}

\author{
Filip Nauwelaerts ${ }^{1}$, Dirk Van Troyen ${ }^{2}$, Guy A. E. Vandenbosch ${ }^{3}$ \\ ${ }^{1}$ Laboratoria De Nayer v.z.w., J. P. De Nayerlaan, St-Katelijne Waver, Belgium; ${ }^{2}$ Department E-ICT, Lessius Hogeschool/ Campus \\ De Nayer ass. K. U. Leuven, J. P. De Nayerlaan, St-Katelijne Waver, Belgium; ${ }^{3}$ Department of Electrical Engineering, K. U. Leuven, \\ Leuven, Belgium. \\ Email: f.nauwelaerts@labodenayer.be
}

Received January $26^{\text {th }}, 2010$; revised June $2^{\text {nd }}, 2010$; accepted June $10^{\text {th }}, 2010$.

\begin{abstract}
This paper summarizes a theoretical design analysis for the implementation of an electromagnetic modeling tool, focusing on the simulation of electromagnetic field propagation inside reverberation chambers. The simulation algorithms which have been developed rely on a ray tracing technique, adapted in such a way as to maximize compatibility with the specific requirements and parameters, as applicable for reverberation chambers. The most significant example of a typical parameter is the high rate of wave reflections inside the chambers' cavity. An implementation of the algorithms was used for simulation of several theoretically predictable cases. Study of the results of these test cases showed that some of the design decisions and algorithms used need to be reviewed in order to optimize the computational aspects of the application, such as resource management (memory, CPU time). Results of typical sample cases are re- viewed in this paper as well, in order to identify possible pitfalls and objectives for future research.
\end{abstract}

Keywords: Electromagnetic Modeling, Computational Electromagnetics, Ray Tracing

\section{Introduction}

Depending on its intended domain of application, electromagnetic immunity of an electronic device towards radiated electromagnetic fields can be demonstrated by using a reverberation chamber $[1,2]$. Typically, the internal chamber cavity consists of highly conductive material, which leads to a high degree of electromagnetic wave reflections. In combination with a tunable mode stirrer, standing wave patterns are created, resulting in high field strengths on a broad frequency spectrum.

Looking at radiated immunity assessments according to IEC61000-4-21, usage of a reverberation chamber is not allowed unless certain criteria are met. For example, during an immunity assessment, the device under test needs to be placed inside a rectangular virtual volume, where it is required that a field homogeneity is guaranteed within this volume, with the device put in place. This field homogeneity criterion states that the field strength amplitude, measured at the eight corner points of the virtual volume (and for each polarization respectively), lies within a specified standard deviation from the normalized mean value of the normalized maximum values obtained at each of these eight locations, during one rotation of the mode stirrer.

This preliminary validation, referred to as a chamber loading verification, consumes a significant amount of measurement time. Results from a ray tracing simulation allow calculation of the electric field vector (amplitude and polarisation at a specified moment in time) at each of the eight corners of the virtual volume. This in turn allows prediction of the influence of a specific device on the chamber loading, without having to cope with the aforementioned time loss.

Moreover, the number of locations for which the resulting field vector is calculated by the adapted ray tracing technique does not have to be limited to the eight corner points. This number can be expanded to cover a specified volume, consisting of a large set of distinct locations. In the obtained simulation model, each of these locations will have a resulting field vector associated with it. This allows representation of the electromagnetic field distribution inside the reverberation chamber as a $3 \mathrm{D}$ vector field. 


\section{Conceptual Description}

Initially, identification and definition of all relevant parameters is required, such as chamber dimensions, source antenna position and orientation with respect to the chamber origin, transmit antenna radiation pattern, conductivity of each enclosure and locations for which a resultant field vector is desired.

These parameters are translated into a set of equations, which allow interpretation by the adapted ray tracing algorithm. The algorithm decomposes the propagated electromagnetic wave front into a finite set of distinct rays. Based on the initial equations, the algorithm calculates how each ray propagates through the defined medium and evaluates possible reflection on obstacle surfaces.

The contribution of each individual ray to the resulting 3D vector field is quantified by implementation of appropriate equations, which allow calculation of the electric field strength amplitude, phase and its polarization for each position on an arbitrary ray at a specified distance from the source.

\section{Basic Ray Tracing Process}

Each individual straight ray path segment has a ray Equation (1) associated with it, which allows calculation of the Cartesian coordinates of an arbitrary point $p$, with $p$ and $p_{0}$ elements of the ray path and $\overrightarrow{\mathrm{u}}$ the unit direction vector.

$$
\mathrm{p}=\mathrm{p}_{0}+\mathrm{s} . \vec{u}
$$

Secondly, it is assumed that each enclosure can be represented by a grouping of one or more rectangular surfaces. For each of these surfaces, plane Equation (2) holds for normal vector and the Cartesian coordinates of an arbitrary point $p$ on the surface. This equation represents the plane (with infinite length and width) which the surface is part of, where $\mathrm{D}$ is a constant that determines part of the position of the plane.

$$
\vec{N} \cdot p=-D
$$

These equations can be used for each enclosure surface and ray, given the initial parameters according to a Cartesian coordinate system. Substitution of Equations (1) and (2) results in Equation (3), which allows evaluation of ray surface intersection.

$$
\overrightarrow{\mathrm{N}} \cdot\left(\mathrm{p}_{0}+\mathrm{s} \cdot \overrightarrow{\mathrm{u}}\right)=-\mathrm{D}
$$

Should this equation hold, then intersection occurs and variable $s$ represents the distance between $\mathrm{p}_{0}$ and the point of intersection, quantifiable by applying Equation (4).

$$
\mathrm{s}=\frac{-\mathrm{D}-\overrightarrow{\mathrm{N}} \cdot \mathrm{p}_{0}}{\overrightarrow{\mathrm{N}} \cdot \overrightarrow{\mathrm{u}}}
$$

Finally, it is verified whether the point of intersection lies within the surfaces boundaries by an inside-outside verification.

Depending on the intersected surface's conductivity, reflection could occur [3], which is considered to be specular. A reflected wave front is in its turn represented by a finite set of reflected rays, for which each ray equation can be calculated based on the law of specular reflection (5), with incident ray direction $\overrightarrow{\mathrm{I}}$ and reflected ray direction $\vec{R}$.

$$
\overrightarrow{\mathrm{R}}=\overrightarrow{\mathrm{I}}-(2 \cdot \overrightarrow{\mathrm{I}} \cdot \overrightarrow{\mathrm{N}}) \cdot \overrightarrow{\mathrm{N}}
$$

This process of reflection continues iteratively for a defined finite number of reflections.

\section{An Adapted Ray Tracing Process}

\subsection{Interference}

With the basic ray tracing process, detection of inference and therefore modeling of standing wave patters is not possible, since phase is not taken into account. This aspect is adapted by implementation of appropriate equations for electromagnetic propagation. This is elaborated into more detail further on.

\subsection{Multiple Viewpoints}

The basic algorithm focuses on representing a 3D scene on a $2 \mathrm{D}$ pixel screen, as observed from one single viewpoint (e.g. the human eye). The viewpoint is used as the starting point for each initial ray, thus the basic process is backtracking the trail through the 3D scene in order to determine each ray's contribution to a pixel on the $2 \mathrm{D}$ screen [4].

Here, a 3D vector field is desired, consisting of a set of field vectors, where each vector is the resultant of contributions from all intersecting rays at one of the specified locations of interest. For this reason it is decided to use the source antenna location as the origin of each initial ray, instead of the location of a viewpoint. This is referred to as the alternative backtracking algorithm further on.

\subsection{Spatial Subdivision and Ray Contribution}

Combination of the alternative backtracking and of the assumption of specular reflection could lead to the situation where a certain location of interest will never be reached by a ray. This is due to the fact that an initial angle step is applicable between each successive ray. This angle step inherently introduces inaccuracy with an incremental behavior, linearly proportional to the distance from the source. As a solution, each location of interest is represented as a cubic cell (Figure 1). This way, the area of reception of each location of interest is no longer a single coordinate in the Cartesian system, but has now been enlarged in such a way that it is represented by a cubic volume instead. The edges of each individual cube have to be smaller with respect to the applicable 


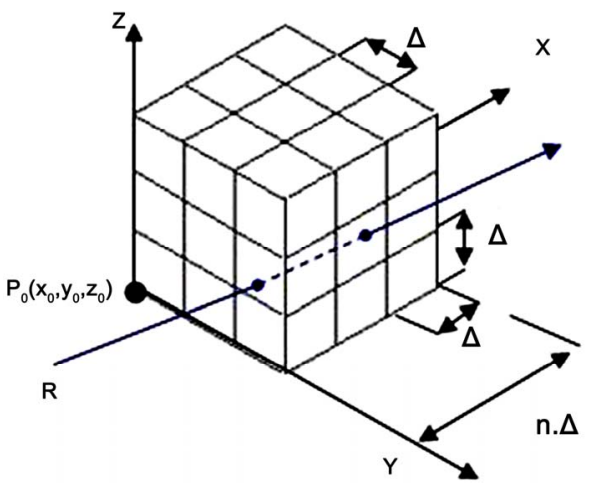

Figure 1. Spatial subdivision

wavelength, otherwise phase information would be lost by this introduced inaccuracy.

The algorithm performs detection of intersection between a ray and a cell facet, based on a set of plane equations for each group of cell facets which are part of the same plane. Identification of the exact cell facet of that group is the result of an inside or outside verification.

To minimize the risk of missing cells, it can be shown that a rule of thumb (Equation (6)) expresses the minimal angular difference $\alpha$ in terms of the number of reflections $n$, the longest chamber dimension $L$ and the cell size $i$.

$$
\alpha=2 \cdot \arcsin \left(\frac{i / 2}{n \cdot L}\right)
$$

As mentioned earlier, modeling at high frequencies requires small cell sizes in turn. This has as a consequence that very small angle steps have to be chosen, which leads to an increasing number of initial rays $r$ and therefore algorithm iterations, since $r \sim 1 / \alpha^{2}$. This has a negative effect on the required processing time

\section{Quantification of Electromagnetic Propa- gation}

\subsection{Correlation with Antenna Radiation Pattern}

Each initial ray originates at the location of the transmit antenna. A 3D antenna radiation pattern allows determination of the initial intensity of the radiated EM field through interpolation, by matching ray direction and azimuth/elevation angle of the radiation pattern. A combination of the radiation pattern of a tuned dipole with a sample initial ray is given in Figure 2.

\subsection{Free Space Field Equations}

For computational convenience, the spherical-polar coordinate system is used to set up equations for the electric field component's amplitude and phase (Figure 3) [5]. Under far field conditions, component $\vec{E}_{r}$ can be neglected.

Using a half wave dipole and assuming free space pro- pagation, Equation (7) is applied to calculate the electric field component $\left|\vec{E}_{\theta}\right|$ at distance $r$ from the transmit antenna.

$$
\left|\vec{E}_{\theta}\right|=j \cdot \frac{Z_{0} \cdot I_{m}}{2 \pi r} \cdot e^{-j \cdot k \cdot r} \cdot\left[\frac{\cos (k \cdot L / 2 \cdot \cos \theta)-\cos (k \cdot L / 2)}{\sin \theta}\right] \text { (7) }
$$

\subsection{Reflection and Polarization}

Each enclosure inside the cavity of the reverberation chamber acts as a discontinuity in the propagation medium. Associating each discontinuity with a reflection coefficient allows calculation of the amount field that is reflected, relative to the incident field.

Reflections will occur differently however for EM waves polarized in parallel or perpendicular to the plane of incidence, leading to two reflection coefficients: $\Gamma_{H}$ and $\Gamma_{\mathrm{V}}$. This has as a consequence that each incident wave needs decomposition into a parallel and a perpendicular component. Their contributions to the total reflected field vector is calculated separately, according to Equation (8).

$$
\vec{E}_{R}=\left|\vec{E}_{H}\right| \cdot \Gamma_{H} \cdot \vec{h}+\left|\vec{E}_{V}\right| \cdot \Gamma_{V} \cdot \vec{v}_{R}
$$

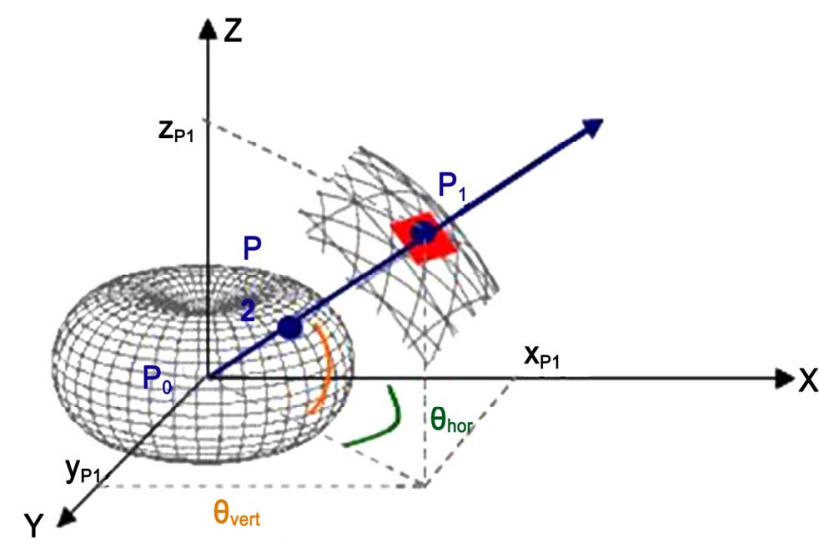

Figure 2. Antenna radiation pattern and ray generation

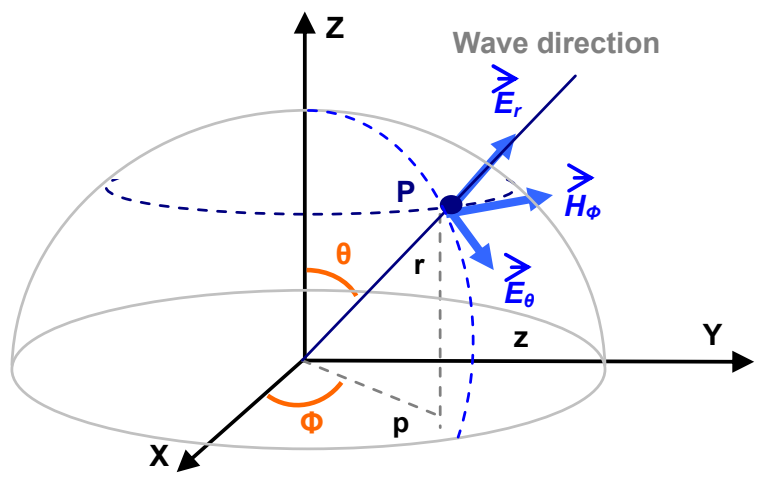

Figure 3. Spherical-polar coordinate system 
$\vec{E}_{H}$ and $\vec{E}_{V}$ represent the parallel, respectively perpendicular components of the incident electric field vector. $\vec{v}_{R}$ is the resultant tangential unit vector after reflection. Equation (9) and Figure 4 show how this new unit vector is calculated.

\section{Validation of Typical cases}

Combination of the adapted ray tracing process design with the equations for EM propagation and reflection, allows implementation of modeling algorithms. A preliminary implementation was used to model several typical cases. A limited number of examples is shown.

\subsection{Fundamental TE10 Mode inside Rectangular Waveguide}

Figure 5 shows the resulting model after simulation of a $\mathrm{TE}_{10}$ mode inside a rectangular waveguide (frequency $=$ $1 \mathrm{GHz}$, width $=\lambda / 2$, height $<<$ width, monopole at $\lambda / 4$ from reflecting front panel). While a $3 \mathrm{D}$ vector field is calculated, only the vertical components' magnitudes are shown for the sake of clarity.

A standing wave pattern can be distinguished along the longitudinal axis, which corresponds to the theoretically expected behavior [6]. The initial part, near the transmitting monopole, shows abrupt changes. These phenomena are considered to be caused by the far field approximations.

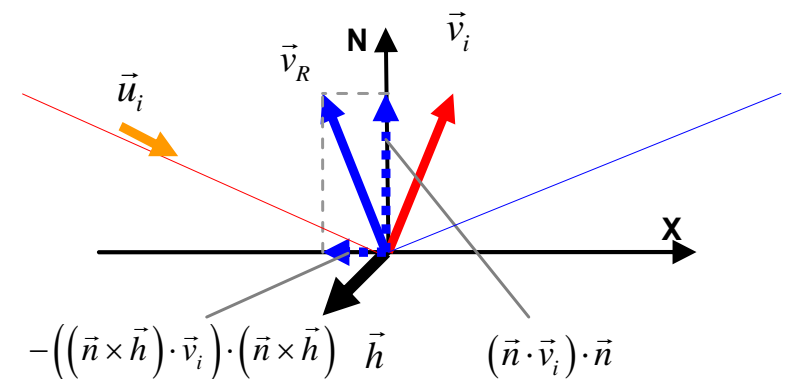

Figrue 4. Antenna radiation pattern and ray generation

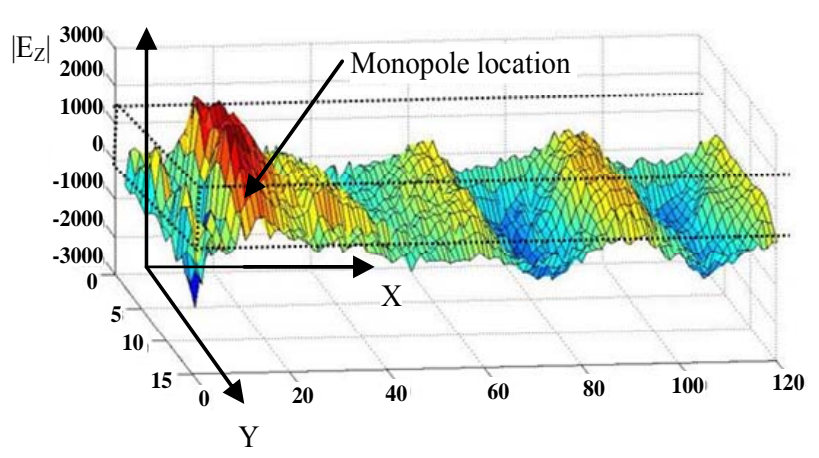

Figure 5. TE10 waveguide model

\subsection{Effect of an Aperture inside a Rectangular Waveguide}

Figure 6 shows the resulting model of an identical waveguide setup, where an additional panel is inserted. This panel is provided with a narrow aperture, where the aperture is dimensioned in such a way that in theory transmission through the waveguide is blocked. The model indeed shows how the standing wave pattern is disrupted by the aperture, where a negligible amount of energy finds its way beyond the discontinuity. Figure 7 shows a top view along the $\mathrm{XY}$-plane.

\subsection{Field Evaluation inside Large Reverberation Chamber}

The effect of chamber loading was modeled. To al-low validation, a measurement was performed with a single field probe located at a specified distance from the transmit antenna (setup 1). By adding cascading absorbers on the floor surface (setup 2), the measured field strength amplitude decreased by $4.77 \mathrm{~dB}$ (Figure 8).

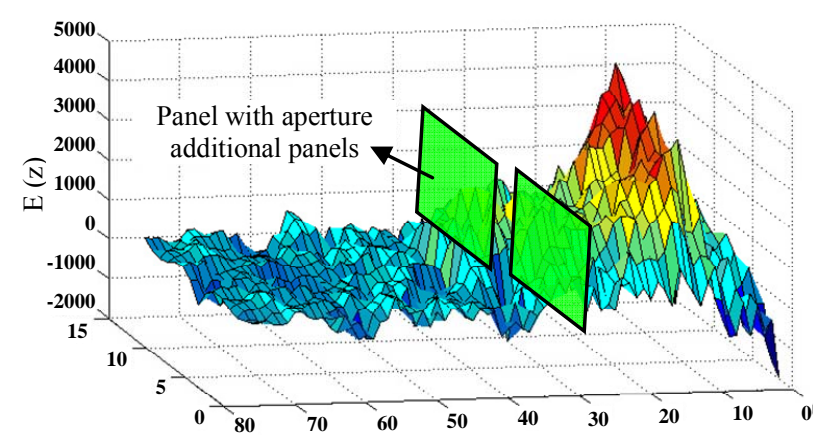

Figure 6. Aperture inside waveguide

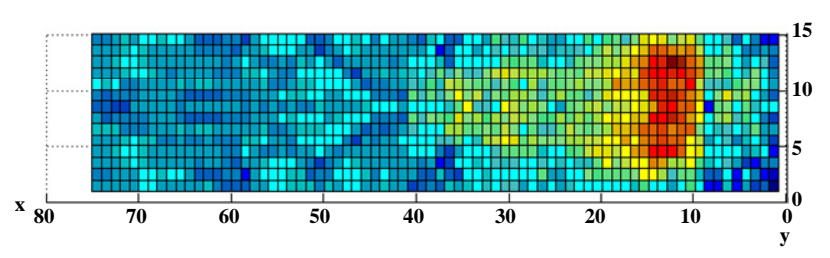

Figure 7. Aperture inside waveguide - top view

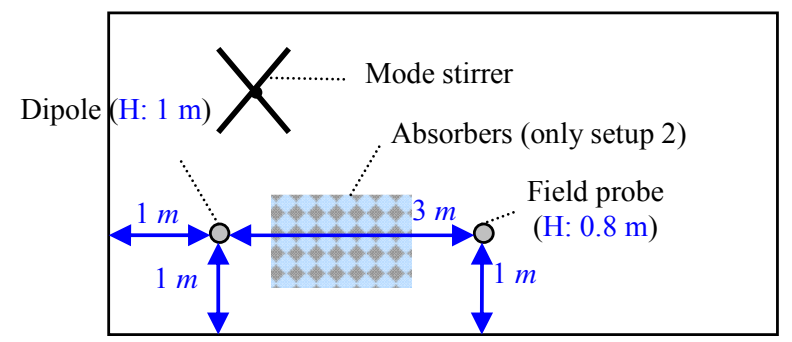

Figure 8. Reverberation chamber (L: 9m, W: 5m, H: 4m) setup 1 and 2 
A correct model should allow derivation of a comparable damping value of $4.77 \mathrm{~dB}$. Both setups were modeled for a set of locations of interest $(15 \times 15 \times 15=$ 3375 locations in total), taking a maximum of 10 reflections into account. The resulting vector fields of both setups are given in Figures 9(a) and 9(b), where each small, colored line represents a single field vector. Each single field vector corresponds to the resultant field vector for a specific location of interest, as calculated by the designed algorithm.

In order to allow numerical analysis of the resulting amplitudes, the values of both models are mapped on a histogram (Figure 10). This histogram shows how many of the calculated field vectors for each setup have an amplitude for which the absolute value lies within one of the specified intervals. Although the values for setup 1 have the tendency to be slightly higher than the values for setup 2, it can't be stated that the differences shown are in correlation with the measurements.

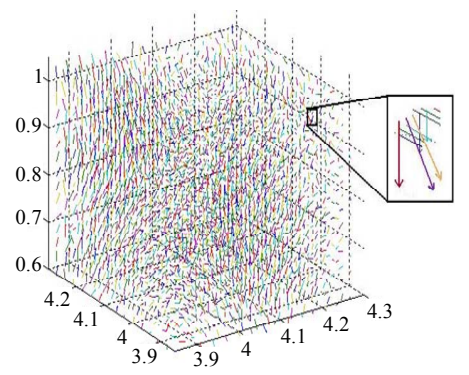

(a)

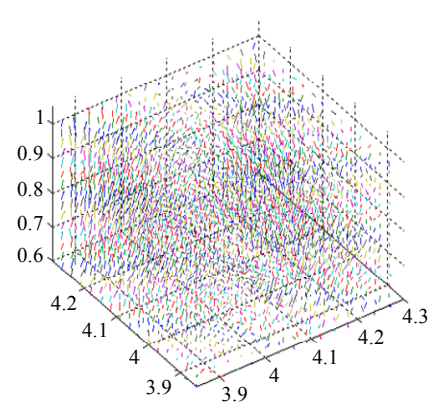

(b)

Figure 9. (a) 3D vector field model of $\vec{E}$ without; (b) $3 D$ vector field model of $\vec{E}$ with absorbers

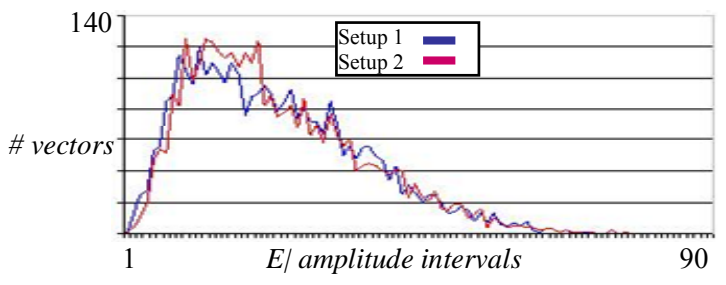

Figure 10. Histogram of field vector amplitudes

\section{Conclusions and Outline for Future Research}

Validation through sample cases show that the current design delivers acceptable results for smaller cavities (e.g. waveguides), but accuracy for larger environments with high reflection rates still remains insufficient (validation of the chamber loading inside a large reverberation chamber showed that a measured attenuation of $4.77 \mathrm{~dB}$ could not be reproduced by interpretation of simulation models).

One of the aspects in the designed algorithm which is highly related to this accuracy is the angular difference between initial rays. Equation (6) already indicated the dependencies between frequency, cell size and their effect on required processing time.

In terms of processing time, it can also be noticed that the current design often relies on goniometric functions. Replacement by matrix transformations for example would improve the resource requirements. A second improvement could be implemented by grouping the surfaces into larger sets during intersection evaluation, and to perform intersection evaluation on these sets first. This should lead to an $\mathrm{O}(\log n)$ search algorithm instead of the current $\mathrm{O}(\mathrm{n})$ version.

For each ray, the total number of reflections was chosen to be 10 for most aforementioned cases, where this number should be related to the remaining field power after attenuation and absorption.

The current design relies on the law of specular reflection. The diffusion is not taken into account, which also leads to less accurate models [7].

These aforementioned conclusions present the outline of further study in the context of a Ph.D. research, where a possible scenario could be the combination of different simulation techniques, such as ray tracing, FEM, BEM, FDM, FDTD, etc.

\section{REFERENCES}

[1] EUROCAE - "Environmental conditions and test procedures for airborne equipment," RTCA DO-160D, RTCA, Washington, DC, 1997.

[2] IEC 61000-4-21, IEC Central Office, Switzerland, 2000.

[3] B. de Greve, "Reflections and Refractions in Ray Tracing," 2007. http://www.bramz.net/data/writings/reflection transmission.pdf

[4] D. Heam and P. Baker, Computer Graphics with OpenGL. Pearson Prentice Hall, New Jersey, 2003.

[5] J. R. Wait, "Introduction to Antennas \& Propagation," Short Run Press Ltd, England, 1986.

[6] A. W. Cross, "Experimental Microwaves," Sanders, Stevenage, 1965.

[7] C. M. Goral, K. E. Torrance, D. P. Greenberg and B. Bataille, "Modeling the Interaction of Light Between Diffuse Surfaces," ACM SIGGRAPH Computer Graphics, Vol. 18, No. 3, pp. 213-222, 1984. 